\title{
Detection of Chaos in Some Local Regions of Phase Portraits Using Shannon Entropies
}

\author{
BINGO WING-KUEN LING, CHARLOTTE YUK-FAN HO and PETER KWONG-SHUN TAM \\ Department of Electronic and Information Engineering \\ The Hong Kong Polytechnic University \\ Hung Hom, Kowloon, Hong Kong, China
}

\begin{abstract}
This letter demonstrates the use of Shannon entropies to detect chaos exhibited in some local regions on the phase portraits. When both the eigenvalues of the secondorder digital filters with two's complement arithmetic are outside the unit circle, the Shannon entropies of the state variables are independent of the initial conditions and the filter parameters, except for some special values of the filter parameters. At these special values, the Shannon entropies of the state variables are relatively small. The state trajectories corresponding to these filter parameters either exhibit random-like chaotic behaviors in some local regions or converge to some fixed points on the phase portraits. Hence, by measuring the Shannon entropies of the state variables, these special state trajectory patterns can be detected. For completeness, we extend the investigation to the case when the eigenvalues of the second-order digital filters with two's complement arithmetic are complex and inside or on the unit circle. It is found that the Shannon entropies of the symbolic sequences for the type II trajectories may be higher than that for the type III trajectories, even though the symbolic sequences of the type II trajectories are periodic and have limit cycle behaviors, while that of the type III trajectories are aperiodic and have chaotic behaviors.
\end{abstract}

Keywords: second-order digital filter; two's complement arithmetic; Shannon entropy

\section{Introduction}


It is well known that chaotic behaviors may occur in the second-order digital filters with two's complement arithmetic [Chua, 1988, 1990a; Galias, 1992; Wu, 1993; Kocarev, 1993, 1996; Yu, 2001; Ling, 2003b, 2003e, 2003f, 2003g, 2003h, 2003i], saturation-type arithmetic [Galias, 1990; Ling 2003c] and quantization-type arithmetic [Lin, 1991; Ling, 2003a]. Similar behaviors may also occur in third-order digital filters with two's complement arithmetic [Chua, 1990b; Ling, 2002, 2003d].

To analyze the chaotic behaviors of the second-order digital filters with two's complement arithmetic, the periodicity and admissibility of the symbolic sequences were investigated [Chua, 1988, 1990a; Galias, 1992; Wu, 1993; Kocarev, 1993, 1996; Yu, 2001; Ling, 2003d, 2003e, 2003f, 2003g, 2003h, 2003i].

However, the effects of the state trajectory behaviors at different filter parameters on the statistical properties of the symbolic sequences and the state variables of these systems have not yet been investigated. Since the state variables will converge to zero and no overflow will occur if both the eigenvalues of the secondorder digital filters with two's complement arithmetic are real and inside the unit circle, the Shannon entropies of the symbolic sequences are therefore zero. When the eigenvalues are complex and inside or on the unit circle, three types of trajectories are exhibited on the phase portraits [Chua, 1988, 1990a; Galias, 1992; Wu, 1993; Kocarev, 1993, 1996; Yu, 2001; Ling, 2002, 2003a, 2003d, 2003e, 2003g, 2003h]. The type of trajectories depends on the filter parameters and the initial conditions of the filters. It is found that the symbolic sequences are constant if the system exhibits the type I trajectory. Hence, the Shannon entropies of the symbolic sequences are zero for the type I trajectory. But what are the related results for the type II and type III trajectories? Since the type III trajectory exhibits chaotic fractal patterns on the phase portraits and the symbolic sequences are aperiodic, in contrast to the type II trajectory which only exhibits limit cycle behaviors and the symbolic sequences are periodic, one may expect that the Shannon entropies of the symbolic sequences for the type III trajectory may be higher than that for the type II trajectory. But is this intuitive implication true? Moreover, it is well known that when both the eigenvalues are outside the unit circle, random-like chaotic patterns are typically exhibited all over the phase portraits. One may ask: are there any exceptional cases? If yes, what are the Shannon entropies of the state variables at these filter parameter values? What features will such phase portraits exhibit? 
The organization of this letter is shown as the following. The system is first described in section 2, followed by the simulation results in section 3 . Finally, a conclusion is given in section 4 .

\section{System description}

Assume a second-order digital filter with two's complement arithmetic is realized in direct form. The system block diagram is shown in figure 1 . The state space model of the overall system can be represented as follows:

$\mathbf{x}(k+1) \equiv\left[\begin{array}{l}x_{1}(k+1) \\ x_{2}(k+1)\end{array}\right]=\left[\begin{array}{c}x_{2}(k) \\ f\left(b \cdot x_{1}(k)+a \cdot x_{2}(k)\right)\end{array}\right]$ for $k \geq 0$

where $a$ and $b$ are the filter parameters; $x_{1}(k)$ and $x_{2}(k)$ are the state variables; and $f$ is the nonlinearity due to the use of two's complement arithmetic.

The nonlinear function $f$ can be modeled as:

$f(v)=v-2 \cdot n$ such that $2 \cdot n-1 \leq v<2 \cdot n+1$ and $n \in \mathbf{Z}$

Hence, the state space model of the overall system can be represented as:

$$
\begin{aligned}
& \mathbf{x}(k+1)=\left[\begin{array}{c}
x_{2}(k) \\
b \cdot x_{1}(k)+a \cdot x_{2}(k)+2 \cdot s(k)
\end{array}\right] \\
& =\mathbf{A} \cdot \mathbf{x}(k)+\left[\begin{array}{l}
0 \\
2
\end{array}\right] \cdot s(k) \text { for } k \geq 0
\end{aligned}
$$

where $\left[\begin{array}{l}x_{1}(k) \\ x_{2}(k)\end{array}\right] \in I^{2} \equiv\left\{\left[\begin{array}{l}x_{1}(k) \\ x_{2}(k)\end{array}\right]:-1 \leq x_{1}(k)<1,-1 \leq x_{2}(k)<1\right\}$ for $k \geq 0$

$$
\mathbf{A} \equiv\left[\begin{array}{ll}
0 & 1 \\
b & a
\end{array}\right]
$$

and $s(k) \in\{-m, \cdots,-1,0,1, \cdots, m\}$ for $k \geq 0$

in which $m$ is the minimum integer satisfying

$-2 \cdot m-1 \leq b \cdot x_{1}(k)+a \cdot x_{2}(k)<2 \cdot m+1$ for $k \geq 0$ 


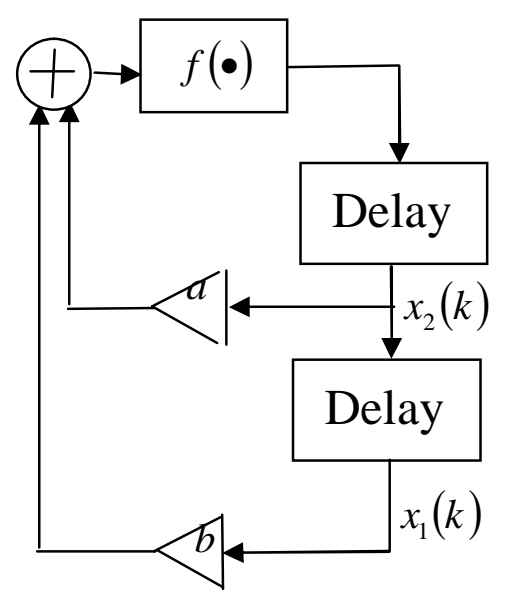

Figure 1: A second-order digital filter with two's complement arithmetic

\section{Simulation results}

When both the eigenvalues of the second-order digital filters with two's complement arithmetic are outside the unit circle, we found that the Shannon entropies of the state variables are independent of the initial conditions and the filter parameters, except for some special values of $a$ and $b$. At these special values of $a$ and $b$, the Shannon entropies of the state variables are relatively small. To demonstrate this phenomenon, initial conditions are generated randomly and the Shannon entropies of the state variables are shown in figure 2 .

One may expect that there are random-like chaotic patterns exhibited all over the phase portraits if both the eigenvalues of the second-order digital filters with two's complement arithmetic are outside the unit circle, as shown in figure 3a. What features will the phase portraits exhibit if the filters are operating at these special filter parameter values? We conducted extensive simulations and found that the state trajectories either exhibit random-like chaotic behaviors in some local regions or converge to some fixed points on the phase portraits, as shown in figure $3 \mathrm{~b}$ and $3 \mathrm{c}$, respectively. 


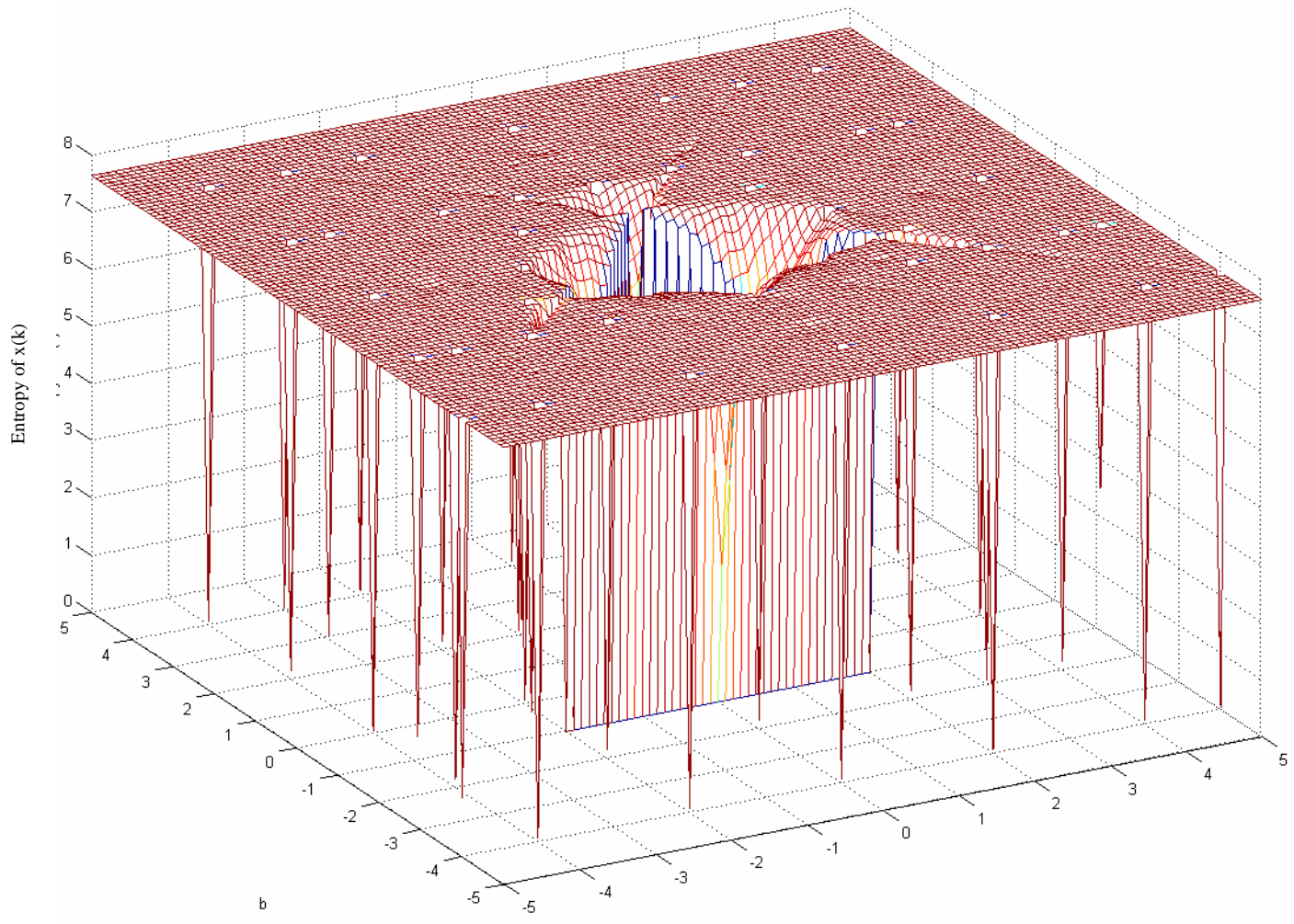

Figure 2: Shannon entropies of the state variables at different filter parameters when

$$
\mathbf{x}(0)=\left[\begin{array}{c}
0.9003 \\
-0.5377
\end{array}\right] \text {. }
$$

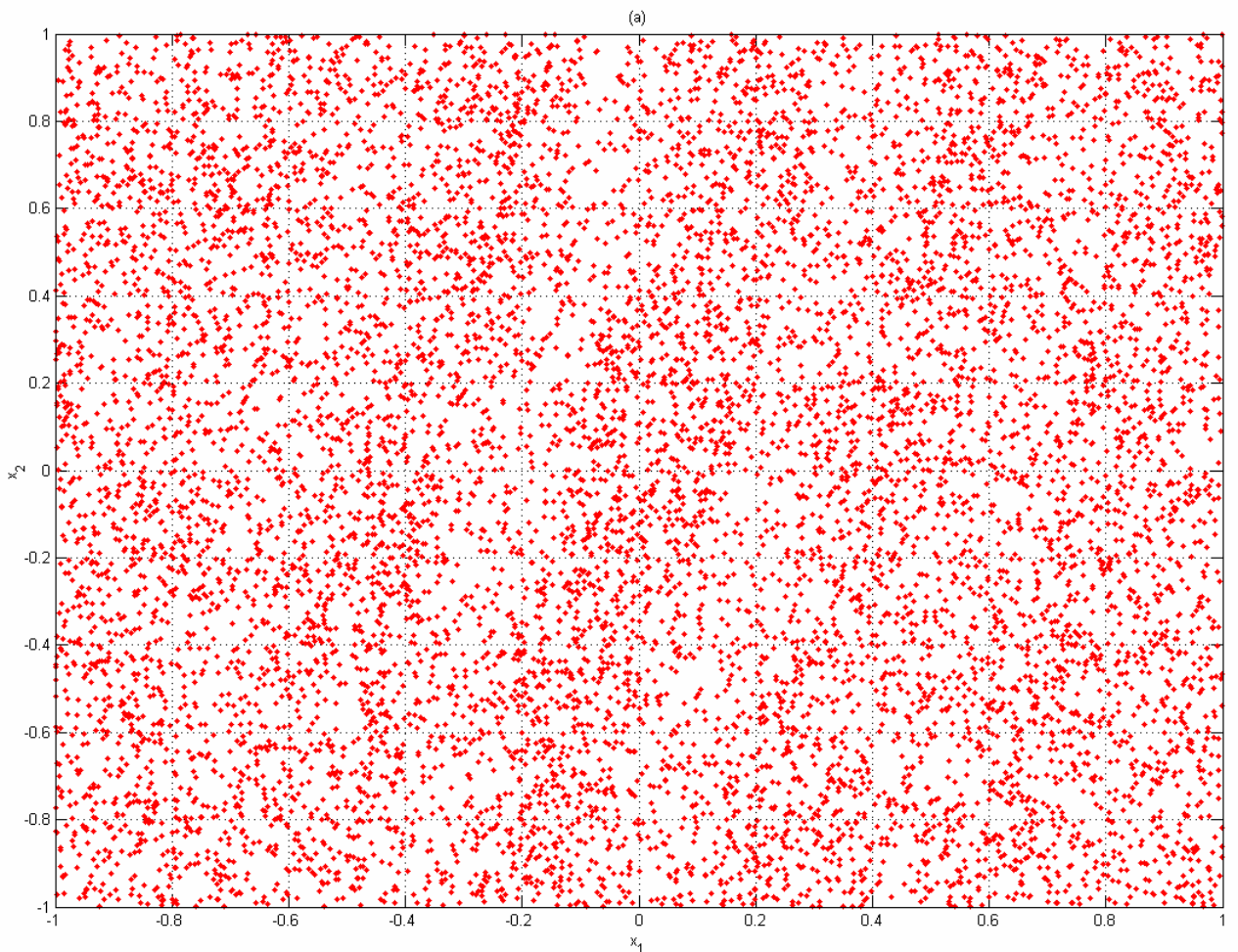

Figure 3: (a) Phase portraits of a second-order digital filter with two's complement arithmetic when $\mathbf{x}(0)=\left[\begin{array}{c}0.9003 \\ -0.5377\end{array}\right], a=4.2$ and $b=4.6$. 


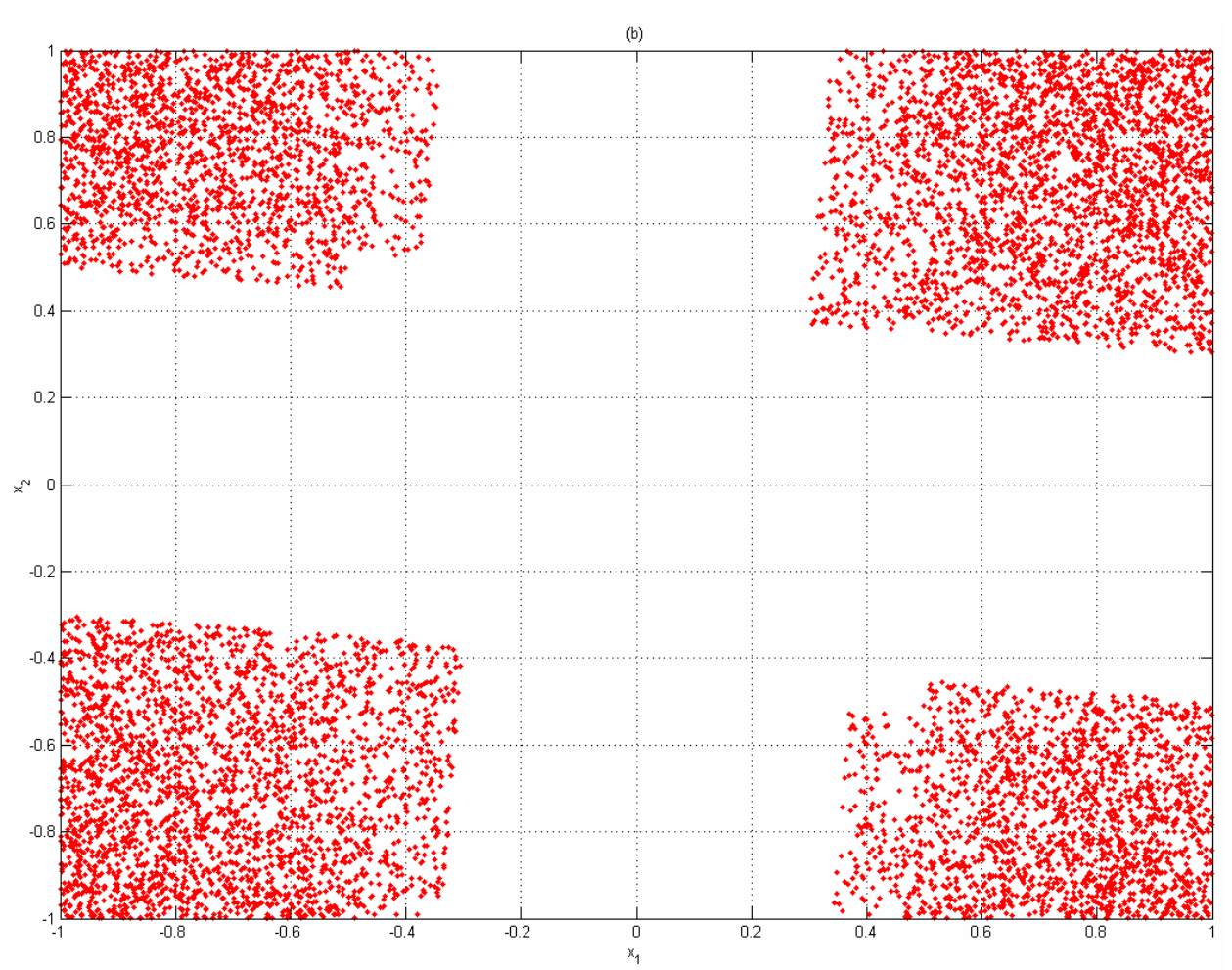

Figure 3: (b) Phase portraits of a second-order digital filter with two's complement arithmetic when $\mathbf{x}(0)=\left[\begin{array}{c}0.9003 \\ -0.5377\end{array}\right], a=-0.1$ and $b=-1.6$.
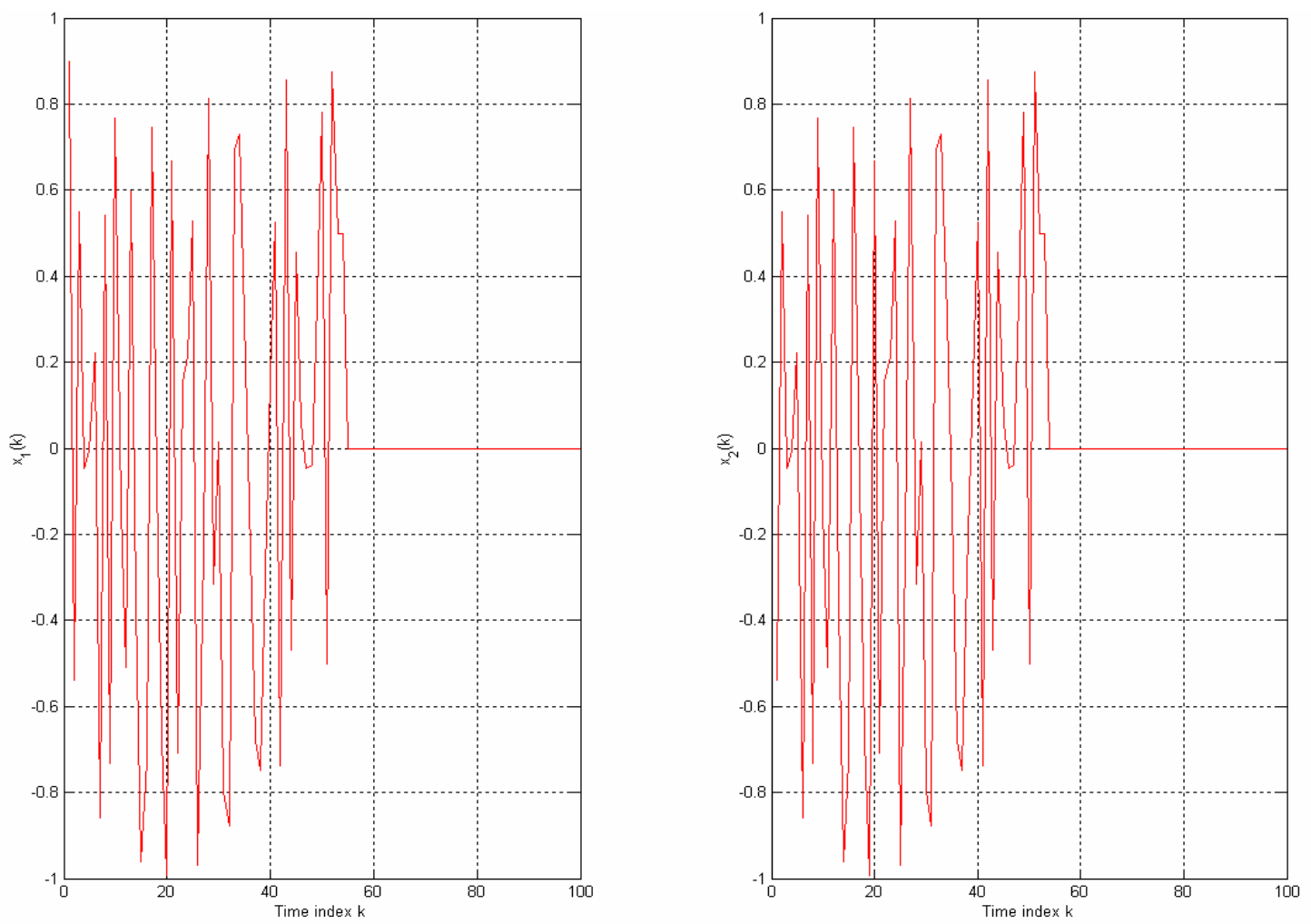

Figure 3: (c) State trajectories of a second-order digital filter with two's complement arithmetic when $\mathbf{x}(0)=\left[\begin{array}{c}0.9003 \\ -0.5377\end{array}\right], a=-4$ and $b=-4$. 
When $a$ and $b$ are multiples of 2 , the phase portraits will converge to some fixed points and the Shannon entropies of the state variables are relatively small. To understand this phenomenon, we model the filtering process as the sum of Bernoulli shift operations. Since only a finite number of bits represent a number in the existing digital circuits, the initial condition can be represented in a binary form with a finite number of bits as follows:

$$
\begin{aligned}
& \left|x_{1}(0)\right|=\sum_{n=1}^{N} p_{n} \cdot 2^{-n} \\
& \left|x_{2}(0)\right|=\sum_{n=1}^{N} q_{n} \cdot 2^{-n}
\end{aligned}
$$

where $p_{n}, q_{n} \in\{0,1\}$ for $n=1,2, \cdots, N$

and $N$ is the number of bits, not including the sign bit, for representing a number in these digital circuits.

Since $a$ and $b$ are even numbers, we can let:

$$
\begin{aligned}
& |a|=\sum_{n=1}^{M} \alpha_{n} \cdot 2^{n} \\
& |b|=\sum_{n=1}^{M} \beta_{n} \cdot 2^{n}
\end{aligned}
$$

where $\alpha_{n}, \beta_{n} \in\{0,1\}$ for $n=1,2, \cdots, M$

$\left|b \cdot x_{1}(0)\right|=\left(\sum_{n=1}^{M} \beta_{n} \cdot 2^{n}\right) \cdot\left(\sum_{n=1}^{N} p_{n} \cdot 2^{-n}\right)$

$=s^{\prime}+\sum_{j=1}^{M} \beta_{j} \cdot\left(\sum_{n=j+1}^{N} p_{n} \cdot 2^{j-n}\right)$

where $s^{\prime} \in Z^{+} \cup\{0\}$

Since the summation of $p_{n} \cdot 2^{j-n}$ in $\sum_{n=j+1}^{N} p_{n} \cdot 2^{j-n}$ is from $n=2$ to $n=N$, the most significant bit is absorbed in $s^{\prime}$ after the first iteration, and all the bits will vanish after $N$ iterations. Therefore, the state trajectories will eventually converge to some fixed points on the phase portraits, and no overflow will occur after $N$ iterations and $s(k)=0$ for $k \geq N$. Hence, the Shannon entropies of the state variables for these special filter parameters are relatively small.

For other special values of the filter parameters, such as when $a=-0.1$ and 
$b=-1.6$, we found that there are chaotic state trajectory patterns exhibited in some local regions on the phase plane. The question is: how to figure out these special filter parameter values? We suggest that if the Shannon entropies of the state variables are relatively small, then the corresponding filter parameters may be the special ones that exhibit some special types of state trajectories on the phase plane.

For completeness, we extend the investigation to the second-order digital filters with two's complement arithmetic with their eigenvalues being complex and inside or on the unit circle. Different types of trajectories of the filter at different initial conditions and the Shannon entropies of the symbolic sequences are shown in figures $4 \mathrm{a}$ and $4 \mathrm{~b}$, respectively.

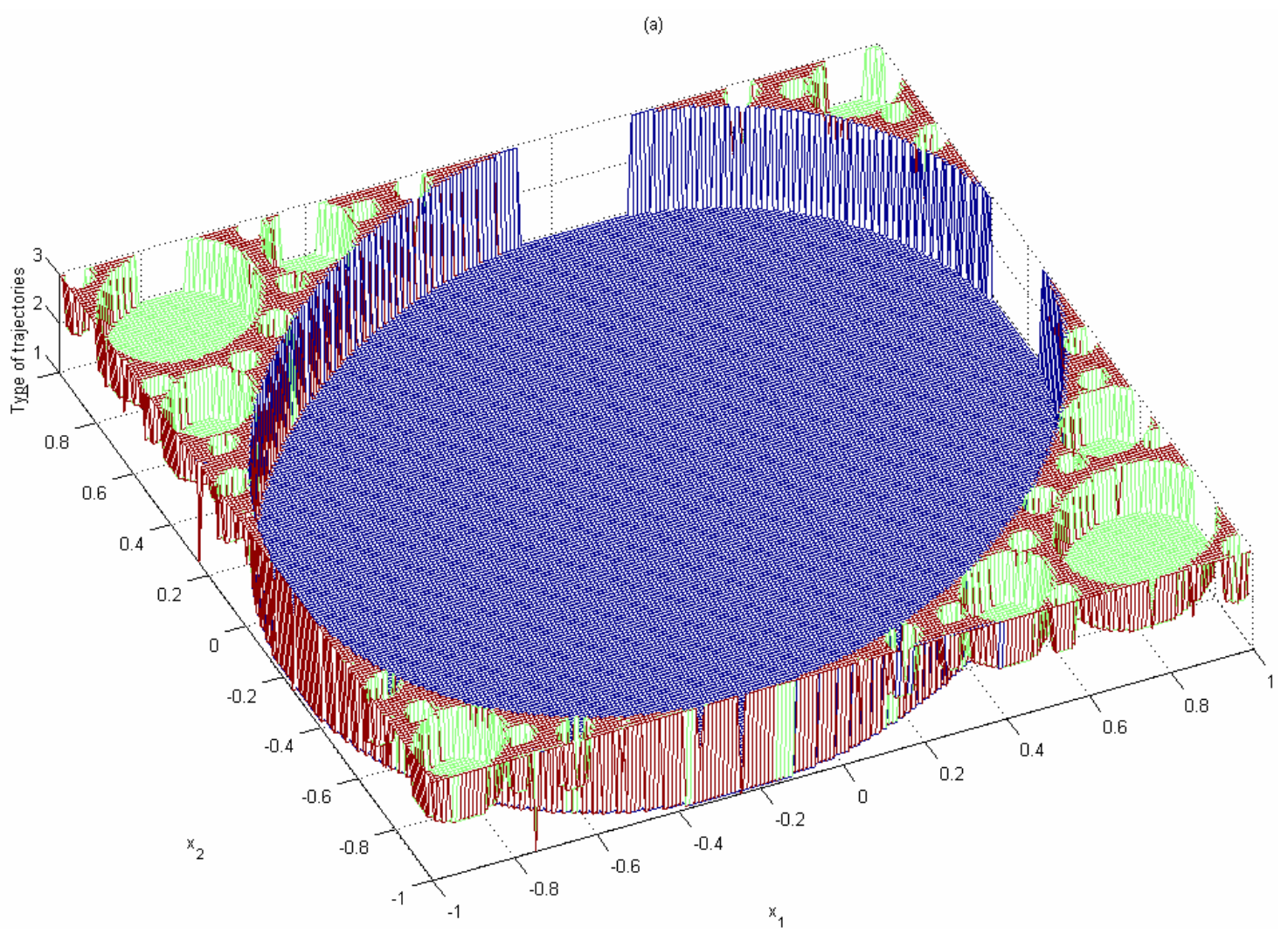




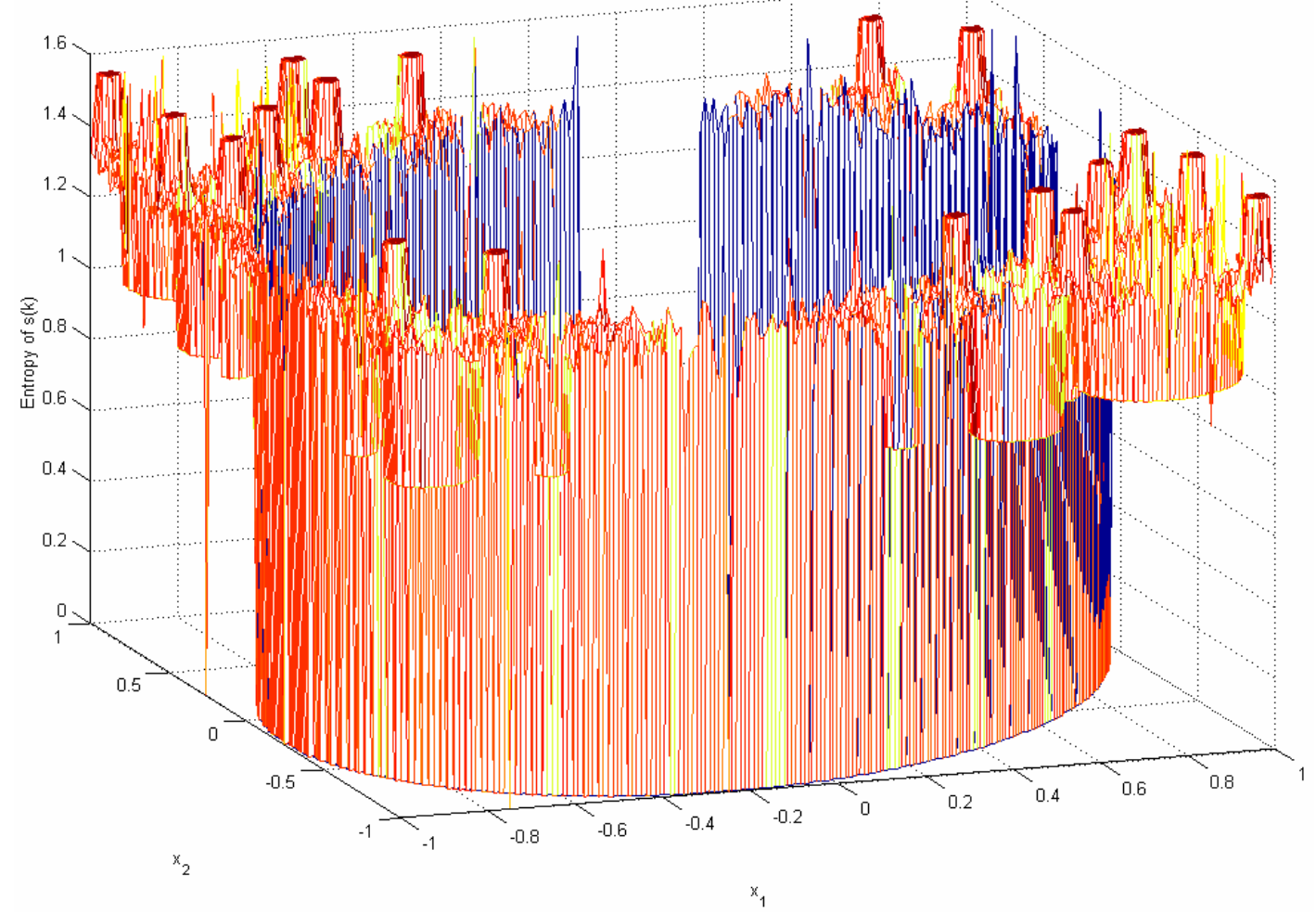

Figure 4: (a) Set of initial conditions for different types of trajectories when $b=-1$ and $a=0.5$. (b) Shannon entropies of symbolic sequences for different initial conditions when $b=-1$ and $a=0.5$.

It can be seen from figure 4 that the Shannon entropies of the symbolic sequences for the type II trajectory may be higher than that for the type III trajectory, even though the symbolic sequences of the type II trajectory are periodic and have limit cycle behaviors, while that of the type III trajectory are aperiodic and have chaotic behaviors. So it is hard to determine the type of trajectories with the Shannon entropies alone, when the eigenvalues are complex and inside or on the unit circle.

\section{Conclusion}

In this letter, we investigated the Shannon entropies of the symbolic sequences and the state variables of the second-order digital filters with two's complement arithmetic for a variety of filter parameters. We conducted extensive simulations and found that when the eigenvalues of the second-order digital filters with two's complement arithmetic are complex and inside or on the unit circle, the Shannon entropies of the symbolic sequences for the type II trajectory may be higher than that for the type III trajectory. When both the eigenvalues of the second-order digital filters with two's complement arithmetic are outside the unit circle, the Shannon entropies of 
the state variables are almost constant and independent of the initial conditions and the filter parameters, except for some special filter parameter values. In these special cases, the state trajectories either exhibit random-like chaotic behaviors in some local regions or converge to fixed points on the phase portraits. In this letter, we suggest to use Shannon entropies to detect these filter parameters.

\section{Acknowledgement}

The work described in this letter was substantially supported partially from a studentship and partially from a research grant (G-YD26) of the Hong Kong Polytechnic University.

\section{References}

[1] Chua, L. O. \& Lin, T. [1988], "Chaos in digital filters," IEEE Transactions on Circuits and Systems, vol. 35, no. 6, pp. 648-658.

[2] Chua, L. O. \& Lin, T. [1990a], "Fractal pattern of second-order non-linear digital filters: a new symbolic analysis," International Journal of Circuit Theory and Applications, vol. 18, pp. 541-550.

[3] Chua, L. O. \& Lin, T. [1990b], "Chaos and fractals from third-order digital filters," International Journal of Circuit Theory and Applications, vol. 18, pp. 241-255.

[4] Galias, Z. \& Ogorzalek, M. J. [1990], "Bifurcation phenomena in second-order digital filter with saturation-type adder overflow characteristic," IEEE Transactions on Circuits and Systems, vol. 37, no. 8, pp. 1068-1070.

[5] Lin, T. \& Chua, L. O. [1991], "On chaos of digital filters in the real world," IEEE Transactions on Circuits and Systems, vol. 38, no. 5, pp. 557-558.

[6] Galias, Z. \& Ogorzalek, M. J. [1992], "On symbolic dynamics of a chaotic secondorder digital filter," International Journal of Circuit Theory and Applications, vol. 20, pp. 401-409.

[7] Wu, C. W. \& Chua, L. O. [1993], "Properties of admissible symbolic sequences in a second-order digital filter with overflow non-linearity," International Journal of Circuit Theory and Applications, vol. 21, pp. 299-307.

[8] Kocarev, L. \& Chua, L. O. [1993], "On chaos in digital filters: case $b=-1$," IEEE Transactions on Circuits and Systems-II: Analog and Digital Signal Processing, 
vol. 40, no. 6, pp. 404-407.

[9] Kocarev, L., Wu, C. W. \& Chua, L. O. [1996], "Complex behavior in digital filters with overflow nonlinearity: analytical results," IEEE Transactions on Circuits and Systems-II: Analog and Digital Signal Processing, vol. 43, no. 3, pp. 234-246.

[10] Yu, X. \& Galias, Z. [2001], "Periodic behaviors in a digital filter with two's complement arithmetic," IEEE Transactions on Circuits and Systems-II: Analog and Digital Signal Processing, vol. 48, no. 10, pp. 1177-1190.

[11]Ling, B. W. K., Chan, A. Y. P., Wong, T. P. L. \& Tam, P. K. S. [2002], "Autonomous response of a third-order digital filter with two's complement arithmetic realized in parallel form," Communications in Information and Systems, vol. 2, no. 4, pp. 435-454.

[12]Ling, B. W. K., Luk, F. C. K. \& Tam, P. K. S. [2003a], "Further investigation on chaos of real digital filters," International Journal of Bifurcation and Chaos, vol. 13, no. 2, pp. 493-495.

[13]Ling, B. W. K. \& Tam, P. K. S. [2003b], "Some new trajectory patterns and periodic behaviors of unstable second-order digital filter with two's complement arithmetic," International Journal of Bifurcation and Chaos, vol. 13, no. 9.

[14]Ling, B. W. K., Hung, W. F. \& Tam, P. K. S. [2003c], "Oscillation and convergence behaviors exhibited in an 'unstable' second-order digital filter with saturation-type nonlinearity," submitted to International Journal of Circuit Theory and Applications.

[15]Ling, B. W. K., Hung, W. F. \& Tam, P. K. S. [2003d], "Autonomous response of a third-order digital filter with two's complement arithmetic realized in cascade form," under revision in International Journal of Circuit Theory and Applications.

[16]Ling, B. W. K., Hung, W. F. \& Tam, P. K. S. [2003e], "Chaotic behaviors of stable second-order digital filters with two's complement arithmetic," to appear in International Journal of Circuit Theory and Applications.

[17]Ling, B. W. K. \& Tam, P. K. S. [2003f], "New results on periodic symbolic sequences of second order digital filters with two's complement arithmetic," International Journal of Circuit Theory and Applications, vol. 31, no. 4, pp. 407421.

[18]Ling, B. W. K., Tam, P. K. S. \& Yu, X. [2003g], "Step response of a secondorder digital filter with two's complement arithmetic," IEEE Transactions on 
Circuits and Systems-I: Fundamental Theory and Applications, vol. 50, no. 4, pp. 510-522.

[19] Ling, B. W. K. \& Tam, P. K. S. [2003h], "Sinusoidal response of a second-order digital filter with two's complement arithmetic," IEEE Transactions on Circuits and Systems-I: Fundamental Theory and Applications, vol. 50, no. 5, pp. 694698.

[20]Ling, B. W. K., Ho, C. Y. F. \& Tam, P. K. S. [2003i], " Admissibility of Unstable Second-order Digital Filter with Two's Complement Arithmetic," submitted to International Journal of Circuit Theory and Applications. 